\title{
Apparent digestibility of amino acids in feedstuffs used in diets for the Pacific white shrimp, Penaeus vannamei
}

\section{Eficiencia digestiva aparente de aminoácidos en ingredientes utilizados en dietas para camarón blanco del pacífico, Penaeus vannamei}

\author{
David Alonso Villarreal-Cavazos*1, Denis Ricque-Marie ${ }^{1}$, Martha Nieto-López ${ }^{1}$, \\ Mireya Tapia-Salazar ${ }^{1}$, Andreas Lemme ${ }^{2}$, Julián Gamboa-Delgado ${ }^{1}$, Lucia Elizabeth Cruz-Suárez ${ }^{1}$ \\ ${ }^{1}$ Departamento de Ecología, Facultad de Ciencias Biológicas, Universidad Autónoma de Nuevo León, \\ Cd. Universitaria, CP 66450, Monterrey (San Nicolás de los Garza), Nuevo León, Mexico. \\ 2 Evonik Degussa GmbH, Rodenbacher Chaussee 4, D-63457 Hanau (Wolfgang), Germany. \\ * Corresponding author. E-mail: david.villarrealcv@uanl.edu.mx, villarrealcd@hotmail.com
}

\begin{abstract}
Apparent digestibility coefficients (ADC) for dry matter, energy, crude protein (CP), and amino acids (AA) were evaluated for 4 feedstuffs used to feed juvenile white shrimp (Penaeus vannamei): fish meal $(73.5 \% \mathrm{CP})$, potato protein concentrate $(89.3 \% \mathrm{CP})$, brewer's yeast $(42.5 \% \mathrm{CP})$, and crustacean meal $(47.2 \% \mathrm{CP})$. Experimental diets included $30 \%$ of the test ingredient and $69 \%$ of a commercial diet supplemented with $1 \%$ chromium oxide as inert marker. Amino acid contents in the ingredients, experimental diets, leached diets, and feces were analyzed by high-performance liquid chromatography. Nutrient loss in water was highest in the fishmeal diet (25\%). The AA with the highest losses in water were lysine, methionine, and arginine (25\%, 23\%, and 21\%, respectively). The ADC for dry matter oscillated between $79.2 \%$ and $90.6 \%$, for $\mathrm{CP}$ between $78.1 \%$ and $91.8 \%$, and for AA between $75.4 \%$ and $96.6 \%$. In all cases the lower limit corresponded to the meal with potato protein concentrate and the upper limit to the crustacean meal. The ADC for energy fluctuated between $89.1 \%$ and $95.2 \%$, with the lower limit for the crustacean meal and the upper limit for the meal with potato protein concentrate.
\end{abstract}

Key words: digestibility, shrimp, Penaeus vannamei, amino acids.

RESUMEN. Los coeficientes de digestibilidad aparente (CDA) de materia seca, energía, proteína cruda (PC) y aminoácidos (AA) fueron evaluados en 4 ingredientes utilizados para alimentar juveniles de camarón blanco (Penaeus vannamei): harina de pescado (73.5\% PC), concentrado proteico de papa ( $89.3 \%$ PC), levadura de cerveza (42.5\% PC) y harina de crustáceo (47.2\% PC). Las dietas experimentales incluyeron $30 \%$ del ingrediente prueba y $69 \%$ de una dieta comercial suplementada con $1 \%$ de óxido de cromo como marcador inerte. El contenido de aminoácidos en los ingredientes, las dietas formuladas, las dietas lixiviadas y las heces fue analizado por cromatografía líquida de alta resolución. La mayor pérdida de nutrientes en el agua se presentó con la dieta de harina de pescado (25\%). Los AA con mayor pérdida en el agua fueron la lisina, la metionina y la arginina $(25 \%, 23 \%$ y $21 \%$, respectivamente). Los CDA para la materia seca oscilaron entre el $79.2 \%$ y el 90.6\%, para la PC entre el 78.1\% y el 91.8\%, y para los AA entre el 75.4\% y el 96.6\%. En todos los casos el límite inferior correspondió al concentrado proteico de papa y el superior a la harina de crustáceo. El CDA para la energía fluctuó entre el 89.1\% y el 95.2\%, con el límite inferior para la harina de crustáceo y el superior para el concentrado proteico de papa.

Palabras clave: eficiencia digestiva, camarón, Penaeus vannamei, aminoácidos.

\section{INTRODUCTION}

Aquaculture feed production is increasingly challenged by the rising cost and limited production of fish meal, the main ingredient in feeds. In the shrimp farming industry, feed is the most expensive input in the variable costs of production when operating grow-out farms. Today a wide range of alternative feedstuffs have to be considered to reduce the amount of fish meal used in penaeid shrimp feed. These feedstuffs must be well characterized to understand their nutritional quality, which is particularly important in the formulation of efficient (highly digestible), ecologically sustainable (low-polluting), and economically competitive feeds. In particular, the apparent digestibility of nutrients in feedstuffs, namely amino acids, plays a very important role in the nutritional quality of

\section{INTRODUCCIÓN}

La producción de alimentos formulados utilizados en la acuacultura enfrenta crecientes problemas porque estos contienen como principal ingrediente harina de pescado, un recurso finito cuyo precio va en constante aumento y cuya producción es limitada. En la industria camaronícola, el alimento es el rubro más caro de los costos variables de producción en la operación de una granja de engorde. Actualmente, para los piensos destinados para la alimentación de camarones peneidos, es necesario considerar una amplia variedad de ingredientes alternos que ayuden a reducir el nivel de inclusión de harinas de pescado. Estos ingredientes deben estar muy bien caracterizados para conocer su calidad nutrimental, lo cual resulta especialmente crucial 
feeds. The aim of the present study is to evaluate the apparent digestibility efficiency of dry matter, energy, proteins, and amino acids in a reference diet (commercial diet) and in 4 feedstuffs used in shrimp feed: Pilchard meal (Sardinops sagax), potato protein concentrate (Solanum tuberosum), brewer's yeast (Saccharomyces cerevisiae), and crustacean meal (Heterocarpus reedi). Apparent digestibility coefficients were adjusted to account for nutrient losses (nutrient leaching) in seawater.

\section{MATERIALS AND METHODS}

Feedstuffs were obtained from feed mills and/or their distributors. We obtained the fish meal (S. sagax) and potato protein concentrate (S. tuberosum) from Sonora (Mexico), the brewer's yeast (S. cerevisiae) from Baja California (Mexico), and the crustacean meal (H. reedi) from Santiago (Chile). Feeds were prepared following the method described by Villarreal-Cavazos et al. (2017); test ingredients were mixed in a KitchenAid mixer (Benton Harbor, Michigan) for $10 \mathrm{~min}$ while adding warm water (30\%), and then for $15 \mathrm{~min}$ after water was incorporated. A Torrey meat grinder (Monterrey, Mexico) with 1.6-mm holes was used. Manufacturing time was 40 min per kilogram of feed, and manufacturing temperatures reached $75-80^{\circ} \mathrm{C}$. The extruded feed was dried in a ventilated oven at $100^{\circ} \mathrm{C}$ for $8 \mathrm{~min}$ and kept at room temperature for one night before packing. Digestibility of feedstuffs was determined following the method described by Cho and Slinger (1979). Experimental diets contained 30\% of the test ingredient and $70 \%$ of the reference diet (Cruz-Suárez et al. 2009).

The digestibility trial was carried out in a closed recirculating artificial seawater system at the Mariculture Program facilities (Faculty of Biological Sciences, Autonomous University of Nuevo León). Twenty-eight identical, fiberglass aquariums with 120-L capacity and regulated flow rate of $710 \mathrm{~mL} \cdot \mathrm{min}^{-1}$ were used. Tanks were randomly distributed. Each diet was tested in 4 replicate tanks with 24 Peaneus vannamei juveniles $(5.4 \pm 0.1 \mathrm{~g})$ each. Shrimp were fed ad libitum starting with $10 \%$ of total biomass in the tank. Fresh feces were collected $(12 \mathrm{~g})$ from each tank with a siphon, immediately after excretion, over a period of $7 \mathrm{~d}$.

The bromatological composition of feedstuffs, diets, and feces was determined with the following methods: 930.15, 990.03, 942.05, and 962.09B for moisture, crude protein, ash, and fiber, respectively (AOAC International 1997). Lipids were extracted using the Soxhlet method (Tecator 1983), and the nitrogen free extract was calculated as the difference. Nutrient loss in seawater was determined according to the method reported by Tapia-Salazar et al. (2012). Chrome content was determined according to the method described by Bolin et al. (1952) and modified by Cruz-Suárez et al. (2009). Amino acid composition was determined according to Llames and Fontaine (1994) and Fontaine (2003). The apparent digestibility coefficients (ADCs) for dry matter, protein, para la formulación de dietas eficientes (altamente digestibles), ecológicamente sustentables (poco contaminantes) y económicamente competentes. En particular, la digestibilidad aparente de nutrientes en los ingredientes, específicamente la de aminoácidos, juega un papel muy importante en la calidad nutrimental de la dieta. El presente estudio tiene por objetivo evaluar la eficiencia de la digestibilidad aparente de la materia seca, la energía, las proteínas y los aminoácidos en una dieta de referencia (dieta comercial) y en 4 ingredientes de alimento de camarón: harina de anchoveta (Sardinops sagax), concentrado proteico de papa (Solanum tuberosum), levadura de cerveza (Saccharomyces cerevisiae) y harina de crustáceo (Heterocarpus reedi). Para el cálculo de los coeficientes de digestibilidad aparente, se contempla un ajuste asociado a las pérdidas de nutrientes (lixiviación de nutrientes) de la dieta en el agua marina.

\section{MATERiales y Métodos}

Los ingredientes evaluados fueron obtenidos de plantas de alimento y/o de sus proveedores. La harina de pescado (S. sagax) provino de Sonora (México); el concentrado proteico de papa (S. tuberosum), de Sonora (México); la levadura de cerveza (S. cerevisiae), de Baja California (México); y la harina de crustáceo, (H. reedi) de Santiago (Chile). Las dietas se elaboraron de acuerdo con la metodología descrita por Villarreal-Cavazos et al. (2017), la cual consiste en mezclar los ingredientes prueba en una mezcladora KitchenAid por 10 min agregando agua tibia (30\%) (Benton Harbor, Michigan) y luego mezclar por $15 \mathrm{~min}$ después de la incorporación del agua. Se utilizó un molino de carne Torrey (Monterrey, México) con un dado con perforaciones de $1.6 \mathrm{~mm}$ de diámetro. El tiempo de procesado fue de 40 min por kilogramo de dieta, y las temperaturas alcanzadas fueron de $75-80^{\circ} \mathrm{C}$. Los extruidos fueron secados en un horno ventilado a $100{ }^{\circ} \mathrm{C}$ por 8 min y permanecieron a temperatura ambiente durante una noche antes de ser empacados. La digestibilidad de los ingredientes fue determinada siguiendo la metodología descrita por Cho y Slinger (1979). Las dietas experimentales contenían 30\% del ingrediente prueba y $70 \%$ de una dieta de referencia (Cruz-Suárez et al. 2009).

El ensayo de digestibilidad se realizó en un sistema cerrado de recirculación de agua marina sintética, en las instalaciones del Programa Maricultura (Facultad de Ciencias Biológicas, Universidad Autónoma de Nuevo León). Se utilizaron 28 réplicas de acuarios de fibra de vidrio de $120 \mathrm{~L}$ regulados a un flujo continuo de $710 \mathrm{~mL}$ de agua por minuto. Los tanques fueron distribuidos en un diseño completamente al azar. Cada dieta experimental fue evaluada en 4 réplicas de acuarios con 24 juveniles $(5.4 \pm 0.1 \mathrm{~g})$ de Peaneus vannamei. Los camarones fueron alimentados ad libitum iniciando con el $10 \%$ de la biomasa total en el acuario. Las heces frescas fueron recolectadas ( $12 \mathrm{~g}$ ) de cada acuario con un sifón durante $7 \mathrm{~d}$ inmediatamente después de haber sido excretadas. 
and amino acids in the diets were calculated using the following equation: \%ADC $\mathrm{Adet}_{\text {diet }}=100-\left[100\left(\mathrm{C}_{\text {diet }} / \mathrm{N}_{\text {diet }}\right) \times\left(\mathrm{N}_{\text {feces }} /\right.\right.$ $\mathrm{C}_{\text {feces }}$ )], where $\mathrm{C}$ and $\mathrm{N}$ are, respectively, the chromium oxide and nutrient concentrations in the diets or feces (dry weight basis). The ADCs for feedstuffs were calculated according to Bureau and Hua (2006). The ADCs for diets and feedstuffs were adjusted considering the percentage of nutrient loss in water due to leaching (Villarreal-Cavazos et al. 2014). We used the leaching values that were obtained after 1-h immersions of feeds in seawater. To account for losses in dietary dry matter, protein, and amino acids before ingestion by the animal, the ADC for each nutrient was corrected for using the equations reported by Nieto-López et al. (2011). Nutrient concentration values (feedstuffs, diets, leached diets, feces) and ADCs were subjected to a one-way analysis of variance and Duncan's multiple range test to separate the treatment averages into normal and homogenous groups. The software used was SPSS v.22 for Windows.

\section{RESULTS}

Crude protein content ranged from $42.5 \%$ to $89.3 \%$. Lipids oscillated between $1.4 \%$ and $10.8 \%$ and crude fiber between $0.1 \%$ and $3.4 \%$. The range for ash content was $5.3-18.1 \%$. Amino acid (AA) proximate composition and AA contents varied greatly between feedstuffs (Table 1). The composition of experimental feeds showed values that were very close to the expected values, according to calculations based on the nutrient concentrations of feedstuffs in the formulated feeds (Table 2). Feeds containing crustacean meal and the fish meal showed the highest nutrient losses $(20 \%$ and $24 \%$, respectively) in seawater (Table 3). The AAs with the highest losses in seawater were lysine, methionine, and arginine $(27 \%, 24 \%$, and $20 \%$, respectively) (Table 3 ).

The ADC varied between $79 \%$ and $91 \%$ for dry matter, between $89 \%$ and $95 \%$ for energy, and between $78 \%$ and $93 \%$ for crude protein. The ADC for total AAs varied between $75 \%$ and $97 \%$ (Table 4). AAs in the crustacean meal feed proved to be highly digestible ( $94.6 \%$ mean digestibility). Digestibility of AAs in the brewer's yeast meal was good (87.1\%). Mean AA digestibility was lowest in the potato protein concentrate $(75.2 \%)$. In general, mean digestibility of AAs in the experimental feedstuffs was more than $90 \%$, except in the case of cystine (80.6\%). The AAs with digestibility values greater than $95 \%$ were methionine, isoleucine, lysine, glycine, and proline. Cystine was the least digestible AA. Corrections for nutrient leaching tended to reduce standard ADC values for most of the experimental feedstuffs (Table 5).

\section{Discussion}

The AA proximate composition and AA contents found for the different feedstuffs were similar to what has been previously reported (NRC 1983, Novus 1996, Hess et al.
La composición bromatológica de los ingredientes, las dietas y las heces fue determinada utilizando los siguientes métodos: para la humedad, el método 930.15; para la proteína cruda, el método 990.03; para las cenizas, el método 942.05; y para la fibra, el método 962.09B (AOAC International 1997). El método Soxhlet fue utilizado para extraer lípidos (Tecator 1983), y el extracto libre de nitrógeno fue calculado por diferencia. La pérdida de nutrientes en agua marina fue estimada de acuerdo con la técnica reportada por Tapia-Salazar et al. (2012). El contenido de cromo fue determinado utilizando el método de Bolin et al. (1952) modificado por Cruz-Suárez et al. (2009). La composición de aminoácidos fue determinada mediante el procedimiento descrito por Llames y Fontaine (1994) y Fontaine (2003). Los coeficientes de digestibilidad aparente (CDA) de materia seca, proteína y aminoácidos de las dietas fueron calculados usando la siguiente ecuación: $\% \mathrm{CDA}_{\text {dieta }}=100-\left[100\left(\mathrm{C}_{\text {dieta }} / \mathrm{N}_{\text {dieta }}\right) \times\left(\mathrm{N}_{\text {heces }} / \mathrm{C}_{\text {heces }}\right)\right]$, donde $\mathrm{C}$ y $\mathrm{N}$ son, respectivamente, las concentraciones del óxido de cromo y del nutriente en dietas o heces (base seca). Los CDA de los ingredientes fueron calculados según Bureau y Hua (2006). Los CDA de las dietas y de los ingredientes fueron ajustados considerando el porcentaje de nutrientes perdidos en el agua por efecto de lixiviación (Villarreal-Cavazos et al. 2014). Se utilizaron los valores de lixiviación obtenidos después de $1 \mathrm{~h}$ de inmersión de las dietas en agua marina. Para tomar en cuenta las pérdidas de materia seca, de proteína y de aminoácidos de las dietas antes de ser ingeridas por el animal, se corrigió el CDA para cada uno de estos nutrientes empleando las ecuaciones reportadas por Nieto-López et al. (2011). Los valores de las concentraciones de nutrientes (ingredientes, dietas, dietas lixiviadas, heces) y los CDA fueron sometidos a un análisis de varianza de una vía y a un análisis múltiple de medias por el método de Duncan para separar las medias de los tratamientos en grupos normales y homogéneos. Se utilizó el paquete computacional SPSS v.22 para Windows.

\section{RESUltados}

El contenido de proteína cruda varió desde $42.5 \%$ hasta $89.3 \%$. Los lípidos oscilaron entre $1.4 \%$ y $10.8 \%$ y la fibra cruda entre $0.1 \%$ y $3.4 \%$. El rango del contenido de cenizas fue 5.3-18.1\%. Tanto la composición proximal como el contenido de aminoácidos (AA) fueron muy variados entre los ingredientes (Tabla 1). La composición de las dietas experimentales mostró valores muy cercanos a los valores esperados, según los cálculos realizados a partir de las concentraciones de nutrientes de los ingredientes utilizados para formular las dietas (Tabla 2). Las dietas con mayor pérdida de nutrientes en el agua fueron la dieta de crustáceo y la dieta de harina de pescado ( $20 \%$ y $24 \%$, respectivamente) (Tabla 3 ). Los AA con mayor perdida en el agua fueron la lisina, la metionina y la arginina $(27 \%, 24 \%$ y $20 \%$, respectivamente) (Tabla 3$)$.

El CDA para la materia seca varió entre $79 \%$ y $91 \%$, para la energía entre $89 \%$ y $95 \%$, y para la proteína cruda entre $78 \%$ y $93 \%$. El CDA para la suma de AA varió entre $75 \%$ y 
Table 1. Proximate composition and amino acid contents for the different experimental feedstuffs.

Tabla 1. Composición proximal y contenido de los aminoácidos de los ingredientes experimentales.

\begin{tabular}{|c|c|c|c|c|}
\hline & FM & PPC & BY & $\mathrm{CM}$ \\
\hline \multicolumn{5}{|c|}{ Proximal composition (\% DM) } \\
\hline Crude protein & 69.7 & 89.3 & 42.5 & 47.2 \\
\hline Crude fat & 10.8 & 1.4 & 3.2 & 3.4 \\
\hline Crude fiber & 0.0 & 0.2 & 2.2 & 3.4 \\
\hline Ash & 18.1 & 5.3 & 6.3 & 13.3 \\
\hline Nitrogen-free extract & 0.0 & 3.2 & 45.0 & 31.3 \\
\hline Gross energy $\left(\mathrm{cal} \cdot \mathrm{g}^{-1}\right)$ & 4,605 & 5,510 & 5,052 & 4,773 \\
\hline \multicolumn{5}{|l|}{ Amino acid profile $(\%)$} \\
\hline Arginine & 4.03 & 4.4 & 2.5 & 2.2 \\
\hline Histidine & 1.87 & 2.0 & 1.0 & 0.9 \\
\hline Isoleucine & 2.62 & 5.0 & 1.7 & 1.7 \\
\hline Leucine & 4.86 & 9.2 & 2.5 & 2.7 \\
\hline Lysine & 5.22 & 7.2 & 2.6 & 2.2 \\
\hline Methionine & 1.80 & 1.9 & 0.6 & 0.8 \\
\hline Phenylalanine & 2.63 & 5.7 & 1.6 & 2.1 \\
\hline Threonine & 2.81 & 5.2 & 1.8 & 1.6 \\
\hline Valine & 3.19 & 6.0 & 2.1 & 2.1 \\
\hline Sum of EAA & 29.0 & 46.7 & 16.3 & 16.3 \\
\hline Alanine & 4.31 & 4.4 & 2.3 & 2.4 \\
\hline Aspartic acid & 6.20 & 10.9 & 3.5 & 3.8 \\
\hline Cystine & 0.58 & 1.2 & 0.5 & 0.4 \\
\hline Glutamic acid & 8.51 & 9.5 & 5.7 & 5.1 \\
\hline Glycine & 4.67 & 4.5 & 1.9 & 2.5 \\
\hline Proline & 3.07 & 4.6 & 2.0 & 1.9 \\
\hline Serine & 2.67 & 4.8 & 1.8 & 1.6 \\
\hline Sum of analyzed AA & 59.10 & 86.4 & 34.2 & 34.0 \\
\hline
\end{tabular}

DM: dry matter; cal: calories; EAA: essential amino acids; AA: amino acids; FM: fish meal; PCC: potato protein concentrate; BY: beer yeast; CM: crustacean meal. 
Table 2. Formula $\left(\mathrm{g} \cdot \mathrm{kg}^{-1}\right.$; dry matter, DM) for experimental diet composition.

Tabla 2. Fórmulas $\left(\mathrm{g} \cdot \mathrm{kg}^{-1}\right.$; materia seca, DM) para la composición de dietas experimentales.

\begin{tabular}{|c|c|c|c|c|c|}
\hline & $\mathrm{RD}$ & FMD & PPCD & BYD & CMD \\
\hline Reference ingredient $^{+}$ & 1,000 & 700 & 700 & 700 & 700 \\
\hline Fish meal & - & 300 & - & - & - \\
\hline Potato protein concéntrate & - & & 300 & - & - \\
\hline Beer yeast & - & & - & 300 & - \\
\hline Crustacean meal & - & & - & - & 300 \\
\hline \multicolumn{6}{|c|}{ Proximal composition (\% DM) } \\
\hline Crude protein & 34.6 & 46.5 & 50.3 & 36.2 & 37.7 \\
\hline Crude fat & 10.6 & 10.5 & 7.8 & 8.3 & 8.4 \\
\hline Crude fiber & 3.7 & 2.1 & 2.6 & 3.3 & 3.7 \\
\hline Ash & 11.0 & 14.5 & 9.4 & 9.7 & 12.0 \\
\hline Nitrogen-free extract & 39.9 & 26.9 & --- & 42.2 & --- \\
\hline Gross energy $\left(\mathrm{cal} \cdot \mathrm{g}^{-1)}\right.$ & 4,750 & 4,162 & 4,949 & 4,811 & 4,728 \\
\hline Arginine & 2.0 & 2.8 & 2.7 & 2.1 & 2.0 \\
\hline Histidine & 0.7 & 1.1 & 1.1 & 0.8 & 0.8 \\
\hline Isoleucine & 1.3 & 1.8 & 2.4 & 1.5 & 1.4 \\
\hline Leucine & 2.3 & 3.2 & 4.3 & 2.5 & 2.4 \\
\hline Lysine & 1.8 & 3.1 & 3.4 & 2.2 & 1.8 \\
\hline Methionine & 0.7 & 1.1 & 1.0 & 0.7 & 0.7 \\
\hline Phenylalanine & 1.6 & 2.0 & 2.7 & 1.6 & 1.7 \\
\hline Threonine & 1.2 & 1.8 & 2.3 & 1.5 & 1.3 \\
\hline Valine & 1.5 & 2.2 & 2.9 & 1.8 & 1.7 \\
\hline Sum of EAA & 13.1 & 19.0 & 22.8 & 14.7 & 13.8 \\
\hline Alanine & 1.8 & 2.7 & 2.5 & 2.1 & 1.9 \\
\hline Aspartic acid & 2.9 & 4.1 & 5.2 & 3.3 & 3.1 \\
\hline Cystine & 0.4 & 0.4 & 0.6 & 0.4 & 0.4 \\
\hline Glutamic acid & 6.0 & 7.1 & 7.0 & 5.7 & 5.7 \\
\hline Glycine & 2.0 & 3.0 & 2.7 & 2.0 & 2.1 \\
\hline Proline & 2.2 & 2.6 & 2.8 & 2.1 & 2.1 \\
\hline Serine & 1.4 & 1.8 & 2.3 & 1.7 & 1.4 \\
\hline Sum of analyzed AA & 29.8 & 40.8 & 45.9 & 32.0 & 30.5 \\
\hline
\end{tabular}

${ }^{+}$Shrimp commercial feed.

RD: reference diet; FMD: fish meal diet; PPCD: potato protein concentrate diet; BYD: beer yeast diet; CMD: crustacean meal diet; cal: calories; EAA: essential amino acids; AA: amino acids. 
2006). In addition, in terms of dry matter, energy, crude protein, and total AAs, all feedstuffs (fish meal, crustacean meal, brewer's yeast, and potato protein concentrate) were more digestible than the reference diet. Goytortúa-Bores et al. (2006) analyzed the crustacean meal (red crab, Pleuroncodes planipes) used to feed Pacific white shrimp and found that ADC values for crude protein were lower than the value observed in the present study $(82-84 \%$ vs $92.8 \%$ ). This value coincides with that reported by Tibbets et al. (2006) for whole krill fed to cod (96\%). Ozório et al. (2012) evaluated the increasing levels of brewer's yeast in tilapia feed and found that apparent digestibility of crude protein decreased $(82.3 \%, 81.3 \%, 78.6 \%, 78.1 \%$, and
97\% (Tabla 4). Los AA de la harina de crustáceo resultaron ser altamente digestibles (digestibilidad promedio del 94.6\%). La digestibilidad de los AA de la harina de levadura de cerveza fue buena (87.1\%), y el concentrado proteico de papa presentó la digestibilidad promedio de AA más baja (75.2\%). En general, la digestibilidad de los AA promedio en los ingredientes evaluados fue mayor que el $90 \%$, con excepción de la cistina $(80.6 \%)$. Los AA que presentaron digestibilidades mayores que el $95 \%$ fueron la metionina, la isoleucina, la lisina, la glicina y la prolina. La cistina fue el AA menos digestible. La corrección por lixiviación de nutrientes para los coeficientes de digestibilidad aparente estándar tendió a reducir los valores en la mayoría de los ingredientes evaluados (Tabla 5).

Table 3. Dietary nutrient losses (percentage of initial content) in seawater.

Tabla 3. Pérdidas de nutrientes dietarios (porcentaje del contenido inicial) en agua marina.

\begin{tabular}{|c|c|c|c|c|c|c|}
\hline & $\mathrm{RD}$ & FMD & PPCD & BYD & CMD & Mean $^{+}$ \\
\hline Dry matter & 14 & 5 & 13 & 11 & 7 & 10 \\
\hline Crude protein & 25 & 25 & 13 & 2 & 20 & $17 \mathrm{~b}$ \\
\hline Arginine & 23 & 19 & 19 & 14 & 24 & $20 \mathrm{c}$ \\
\hline Histidine & 11 & 20 & 16 & 6 & 18 & $14 \mathrm{ab}$ \\
\hline Isoleucine & 13 & 34 & 16 & 3 & 20 & $17 \mathrm{~b}$ \\
\hline Leucine & 9 & 20 & 13 & 4 & 16 & $12 \mathrm{a}$ \\
\hline Lysine & 25 & 42 & 23 & 16 & 29 & $27 \mathrm{e}$ \\
\hline Methionine & 31 & 22 & 25 & 17 & 26 & $24 \mathrm{e}$ \\
\hline Phenylalanine & 12 & 26 & 13 & 4 & 16 & $14 \mathrm{ab}$ \\
\hline Threonine & 11 & 38 & 12 & 4 & 15 & $16 \mathrm{ab}$ \\
\hline Valine & 9 & 21 & 16 & 5 & 20 & $14 \mathrm{ab}$ \\
\hline Sum of EAA & 15 & 10 & 17 & 8 & 20 & $14 \mathrm{ab}$ \\
\hline Alanine & 15 & 19 & 16 & 11 & 22 & $17 \mathrm{~b}$ \\
\hline Aspartic acid & 13 & 25 & 13 & 5 & 15 & $14 \mathrm{ab}$ \\
\hline Cystine & 18 & 22 & 12 & 5 & 11 & $14 \mathrm{ab}$ \\
\hline Glutamic acid & 16 & 20 & 16 & 6 & 16 & $15 \mathrm{ab}$ \\
\hline Glycine & 26 & 32 & 19 & 11 & 25 & $23 \mathrm{c}$ \\
\hline Proline & 23 & 32 & 18 & 10 & 19 & $20 \mathrm{c}$ \\
\hline Serine & 14 & 30 & 12 & 9 & 18 & $17 \mathrm{~b}$ \\
\hline Sum of analyzed AA & 17 & 22 & 16 & 8 & 19 & $16 \mathrm{ab}$ \\
\hline Mean (all AA) & 17 & 24 & 17 & 8 & 20 & $17 \mathrm{~b}$ \\
\hline
\end{tabular}

${ }^{+}$Analysis of variance for AA $(P<0.001)$; different letters indicate significant differences according to Duncan's multiple range test $(\alpha=0.05)$.

RD: reference diet; FMD: fish meal diet; PPCD: potato protein concentrate diet; BYD: beer yeast diet; CMD: crustacean meal diet; EAA: essential amino acids; AA: amino acids. 
$79.4 \%$ ) as inclusion levels of brewer's yeast in the diet increased $(0 \%, 10 \%, 15 \%, 20 \%$, and $30 \%$, respectively). In the present study, for Pacific white shrimp, brewer's yeast was a highly digestible feedstuff $(91.8 \%)$ in terms of crude protein at an inclusion level of $30 \%$. Terrazas-Fierro et al. (2010) reported ADC values for essential AAs in a red crab ( $P$. planipes) meal that were much lower $(82.1 \%)$ than the values found for crustacean meal in the present study (95\%). In the present study, experimental diets were formulated with $30 \%$ of the test ingredient. The Commonwealth Scientific and Industrial Research Organisation (Australia)

\section{DiscuSIÓN}

La composición proximal y la concentración de AA en los diferentes ingredientes evaluados fueron muy similares a lo previamente reportado (NRC 1983, Novus 1996, Hess et al. 2006). Adicionalmente, en términos de materia seca, energía, proteína cruda y AA totales, los ingredientes (harina de pescado, harina de crustáceo, levadura de cerveza y concentrado proteico de papa) fueron más digestibles que la dieta de referencia. Goytortúa-Bores et al. (2006) analizaron la harina de crustáceo (langostilla roja,

Table4. Apparent digestibility coefficients for dry matter, energy, crude protein, and amino acids of ingredients used in Pacific white shrimp (Litopenaeus vannamei) diets.

Tabla 4. Coeficiente de digestibilidad aparente para la materia seca, la energía, la proteína cruda y los aminoácidos de los ingredientes utilizados en dietas para camarones marinos Penaeus vannamei.

\begin{tabular}{|c|c|c|c|c|}
\hline & FM & PPC & BY & $\mathrm{CM}^{+}$ \\
\hline Dry matter & $90 \pm 3 a$ & $79 \pm 1 c$ & $82 \pm 2 b$ & $91 \pm 2 \mathrm{a}$ \\
\hline Energy & $93 \pm 1 b$ & $95 \pm 1 \mathrm{a}$ & $90 \pm 4 c$ & $89 \pm 1 c$ \\
\hline Crude protein & $93 \pm 2 \mathrm{a}$ & $78 \pm 2 d$ & $87 \pm 4 b$ & $92 \pm 2 \mathrm{a}$ \\
\hline Arginine & $98 \pm 1 \mathrm{a}$ & $79 \pm 1 d$ & $92 \pm 4 c$ & $96 \pm 4 b$ \\
\hline Histidine & $97 \pm 1 \mathrm{a}$ & $77 \pm 1 \mathrm{~d}$ & $88 \pm 5 c$ & $92 \pm 4 b$ \\
\hline Isoleucine & $93 \pm 1 \mathrm{a}$ & $75 \pm 1 d$ & $88 \pm 6 c$ & $96 \pm 4 b$ \\
\hline Leucine & $94 \pm 1 \mathrm{a}$ & $75 \pm 1 c$ & $88 \pm 6 b$ & $96 \pm 5 \mathrm{a}$ \\
\hline Lysine & $96 \pm 1 \mathrm{a}$ & $80 \pm 2 c$ & $92 \pm 8 b$ & $96 \pm 6 \mathrm{a}$ \\
\hline Methionine & $99 \pm 1 \mathrm{a}$ & $80 \pm 2 d$ & $90 \pm 6 c$ & $97 \pm 4 b$ \\
\hline Phenylalanine & $93 \pm 1 \mathrm{a}$ & $74 \pm 1 d$ & $87 \pm 5 c$ & $93 \pm 4 b$ \\
\hline Threonine & $99 \pm 1 \mathrm{a}$ & $72 \pm 1 d$ & $84 \pm 6 c$ & $93 \pm 4 . b$ \\
\hline Valine & $97 \pm 1 \mathrm{a}$ & $76 \pm 1 c$ & $90 \pm 6 b$ & $96 \pm 3 \mathrm{a}$ \\
\hline Sum of EAA & $98 \pm 1 \mathrm{a}$ & $76 \pm 1 d$ & $88 \pm 6 c$ & $95 \pm 4 b$ \\
\hline Alanine & $97 \pm 1 \mathrm{a}$ & $76 \pm 1 c$ & $89 \pm 4 b$ & $97 \pm 4 a$ \\
\hline Aspartic acid & $97 \pm 1 \mathrm{a}$ & $73 \pm 1 d$ & $87 \pm 5 c$ & $94 \pm 4 b$ \\
\hline Cystine & $90 \pm 3 a$ & $66 \pm 1 d$ & $73 \pm 6 c$ & $87 \pm 5 b$ \\
\hline Glutamic acid & $97 \pm 1 \mathrm{a}$ & $76 \pm 1 d$ & $88 \pm 4 c$ & $95 \pm 4 b$ \\
\hline Glycine & $89 \pm 2 b$ & $77 \pm 1 \mathrm{c}$ & $89 \pm 3 c$ & $100 \pm 1 \mathrm{a}$ \\
\hline Proline & $97 \pm 1 \mathrm{a}$ & $72 \pm 1 d$ & $85 \pm 5 c$ & $92 \pm 4 b$ \\
\hline Serine & $98 \pm 1 \mathrm{a}$ & $76 \pm 1 c$ & $87 \pm 4 b$ & $97 \pm 3 a$ \\
\hline Sum of analyzed AA & $97 \pm 1 \mathrm{a}$ & $75 \pm 1 c$ & $88 \pm 5 b$ & $95 \pm 4 a$ \\
\hline
\end{tabular}

${ }^{+}$Analysis of variance for AA $(P<0.001)$; different letters indicate significant differences according to Duncan's multiple range test $(\alpha=0.05)$.

FM: fish meal; PPC: potato protein concentrate; BY: beer yeast; CM: crustacean meal; EAA: essential amino acids; AA: amino acids. 
Table 5. Apparent digestibility coefficients (\%) adjusted for nutrient losses in seawater prior to ingestion. Tabla 5. Coeficientes de digestibilidad aparente (\%) ajustados para considerar las pérdidas de nutrientes en agua marina antes de la ingestión.

\begin{tabular}{|c|c|c|c|c|}
\hline & FM & PPC & BY & $\mathrm{CM}^{+}$ \\
\hline Dry matter & $85 \pm 5 b$ & $78 \pm 1 d$ & $82 \pm 3 c$ & $94 \pm 2 \mathrm{a}$ \\
\hline Crude protein & $86 \pm 3 c$ & $77 \pm 2 d$ & $91 \pm 5 b$ & $97 \pm 2 \mathrm{a}$ \\
\hline Arginine & $98 \pm 2 \mathrm{a}$ & $75 \pm 2 c$ & $92 \pm 4 b$ & $94 \pm 5 b$ \\
\hline Histidine & $97 \pm 2 \mathrm{a}$ & $71 \pm 1 \mathrm{c}$ & $88 \pm 5 b$ & $87 \pm 5 b$ \\
\hline Isoleucine & $96 \pm 2 \mathrm{a}$ & $69 \pm 1 c$ & $90 \pm 5 b$ & $92 \pm 5 b$ \\
\hline Leucine & $96 \pm 1 \mathrm{a}$ & $70 \pm 1 d$ & $89 \pm 6 c$ & $92 \pm 5 b$ \\
\hline Lysine & $99 \pm 1 \mathrm{a}$ & $74 \pm 3 c$ & $93 \pm 1 b$ & $93 \pm 1 b$ \\
\hline Methionine & $97 \pm 2 \mathrm{a}$ & $75 \pm 2 c$ & $96 \pm 5 \mathrm{ab}$ & $98 \pm 2 \mathrm{a}$ \\
\hline Phenylalanine & $94 \pm 2 \mathrm{a}$ & $69 \pm 1 c$ & $89 \pm 5 b$ & $91 \pm 5 b$ \\
\hline Threonine & $96 \pm 2 \mathrm{a}$ & $68 \pm 1 d$ & $85 \pm 1 c$ & $91 \pm 2 b$ \\
\hline Valine & $95 \pm 1 \mathrm{a}$ & $71 \pm 1 d$ & $88 \pm 2 c$ & $90 \pm 1 b$ \\
\hline Sum of EAA & $97 \pm 2 \mathrm{a}$ & $71 \pm 2 c$ & $90 \pm 1 b$ & $92 \pm 1 b$ \\
\hline Alanine & $96 \pm 2 \mathrm{a}$ & $71 \pm 2 d$ & $90 \pm 2 c$ & $93 \pm 1 b$ \\
\hline Aspartic acid & $96 \pm 2 \mathrm{a}$ & $69 \pm 1 c$ & $89 \pm 2 b$ & $92 \pm 2 b$ \\
\hline Cystine & $87 \pm 1 b$ & $64 \pm 1 d$ & $81 \pm 2 c$ & $95 \pm 1 \mathrm{a}$ \\
\hline Glutamic acid & $96 \pm 2 \mathrm{a}$ & $72 \pm 2 d$ & $90 \pm 2 c$ & $94 \pm 1 b$ \\
\hline Glycine & $85 \pm 2 b$ & $74 \pm 1 \mathrm{c}$ & $99 \pm 1 \mathrm{a}$ & $99 \pm 1 \mathrm{a}$ \\
\hline Proline & $96 \pm 2 \mathrm{a}$ & $69 \pm 1 c$ & $85 \pm 1 b$ & $88 \pm 1 b$ \\
\hline Serine & $96 \pm 1 \mathrm{a}$ & $71 \pm 1 c$ & $92 \pm 1 b$ & $98 \pm 1 \mathrm{a}$ \\
\hline Sum of analyzed AA & $96 \pm 2 \mathrm{a}$ & $71 \pm 1 d$ & $90 \pm 2 c$ & $93 \pm 2 b$ \\
\hline
\end{tabular}

${ }^{+}$Analysis of variance for AA $(P<0.001)$; different letters indicate significant differences according to Duncan's multiple range test $(\alpha=0.05)$.

FM: fish meal; PPC: potato protein concentrate; BY: beer yeast; CM: crustacean meal; EAA: essential amino acids; AA: amino acids.

work group indicates that the higher the inclusion level of the feedstuff in the experimental diet, the higher the precision in the determination of feedstuff digestibility because differences with the reference diet become clearer and more accurate (Cruz-Suárez et al. 2008). Correction for nutrient leaching tended to increase ADC values for brewer's yeast, whereas the rest of the feedstuffs showed the opposite effect. Tusche et al. (2011) reported low growth rates for rainbow trout when feed contained $50 \%$ of potato protein concentrate. Xie and Jokumsen (1997) reported that growth rates for rainbow trout decreased as inclusion levels of potato protein concentrate increased, which could be associated with low AA digestibility, as shown in the present study for shrimp. The present study showed that the digestive efficiency of
Pleuroncodes planipes) utilizada para alimentar camarón blanco del Pacífico y encontraron valores de CDA para proteína cruda inferiores al valor encontrado en el presente estudio ( $82-84 \%$ vs $92.8 \%$ ). Este valor concuerda con lo reportado por Tibbets et al. (2006) para kril entero alimentado a bacalao (96\%). Ozório et al. (2012) evaluaron niveles crecientes de levadura de cerveza en dietas para tilapia y encontraron que la digestibilidad aparente de proteína cruda disminuyó $(82.3 \%, 81.3 \%, 78.6 \%, 78.1 \%$ y $79.4 \%)$ conforme incrementó el nivel de inclusión de levadura de cerveza en la dieta $(0 \%, 10 \%, 15 \%, 20 \%$ y $30 \%$, respectivamente). En el presente estudio, para el camarón blanco del Pacífico, la levadura de cerveza resultó ser un ingrediente altamente digestible (91.8\%) en términos de proteína cruda 
essential AAs in the experimental fish meal was high (97\%), much higher than the values reported by Yang et al. (2009) (87\%), Terrazas-Fierro et al. (2010) (89\%), and Carvalho et al. 2016 (84\%). This differences could be associated with different factors, such as fish species, raw material (whole fish, by-products resulting from the filleting process, mixture of viscera from other species, etc.), or the elaboration process (drying by direct flame or by steam jacket). However, the previously mentioned studies do not specify on the raw material or elaboration process used.

English translation by Claudia Michel-Villalobos

\section{REFERENCES}

[AOAC International] Association of Official Analytical Chemists International. 1997. Official Methods of Analysis of AOAC International. 16th ed. Cunnift P, editor. Gaithersburg (MD): AOAC International; 1033 pp.

Bolin DW, King RP, Klosterman EW. 1952. A simplified method for the determination of chromic oxide $\mathrm{Cr}_{2} \mathrm{O}_{3}$ when used as an index substance. Science 116(3023): 634-635. https://doi.org/10.1126/science.116.3023.634

Bureau DP, Hua K. 2006. Letter to the editor of Aquaculture. Aquaculture 252: 103-105.

Carvalho RAPLF, Haruo-Ota R, Olveira-Kadry V, Tacon AGJ, Lemos D. 2016. Apparent digestibility of protein, energy and amino acids of six protein sources included at three levels in diets for juvenile white shrimp Litopenaeus vannamei reared in high performance conditions. Aquaculture 465: 223-234. https://doi.org/10.1016/j.aquaculture.2016.09.010

Cho CY, Slinger SJ. 1979. Apparent digestibility measurement in feedstuffs for rainbow trout. In: Halver JE, Tiews K (eds.), Finfish Nutrition and Fishfeed Technology. Vol. 2. Berlin (Germany): Heenemann Verlagsgesellschaft; p. 239-247.

Cruz-Suárez LE, Ricque-Marie D, Tapia-Salazar M. 2008. Integración y análisis global de las metodologías de digestibilidad in vivo. In: Cruz-Suárez LE, Villarreal-Colmenares H, TapiaSalazar M, Nieto-López M, Villarreal-Cavazos DA, RicqueMarie D (eds.), Manual de Metodologías de Digestibilidad in vivo e in vitro para Ingredientes y Dietas para Camarón. Monterrey (Nuevo León; Mexico): Universidad Autónoma de Nuevo León; p. 180-187. ISBN: 978-607-433-020-5.

Cruz-Suárez LE, Tapia-Salazar M, Villarreal-Cavazos D, BeltranRocha J, Nieto-López M, Lemme A, Ricque-Marie D. 2009. Apparent dry matter, energy, protein and amino acid digestibility of four soybean ingredients in white shrimp Litopenaeus vannamei juveniles. Aquaculture 292 (1-2): 87-94. https://doi.org/10.1016/j.aquaculture.2009.03.026

Fontaine J. 2003. Amino acid analysis of feeds. In: D'Mello JPF (ed.), Amino acids in animal nutrition. 2nd ed. Wallingford (United Kingdom): CABI publishing; p. 22-31.

Goytortúa-Bores E, Civera-Cerecedo R, Rocha-Meza S, Green-Yee A. 2006. Partial replacement of red crab (Pleuroncodes planipes) meal for fish meal in practical diets for the white shrimp Litopenaeus vannamei. Effects on growth and in vivo digestibility. Aquaculture 256(1-4): 414-422. https://doi.org/10.1016/j.aquaculture.2006.02.035

Hess V, Fickler J, Fontaine J, Heimbeck W. 2006. AminoDat 3.0Amino acid composition of feedstuffs. Hanau (Germany): Evonik-Degussa GmbH, Health and Nutrition. al 30\% de inclusión. Terrazas-Fierro et al. (2010) reportaron valores de CDA para los AA esenciales en una harina de langostilla roja (P. planipes) muy inferiores (82.1\%) a los encontrados en la harina de crustáceo evaluada en el presente estudio (95\%). El nivel de inclusión del ingrediente en la dieta prueba utilizada en el presente estudio fue del 30\%. El grupo de trabajo del Commonwealth Scientific and Industrial Research Organisation (Australia) menciona que entre mayor sea el nivel de inclusión del ingrediente en la dieta prueba, mayor será la precisión en la determinación de la digestibilidad del ingrediente porque la diferencia con la dieta de referencia se vuelve más clara o más neta (Cruz-Suárez et al. 2008). La corrección por lixiviación tendió a incrementar los valores de CDA para la levadura de cerveza, mientras que para el resto de los ingredientes se observó un efecto contrario. Tusche et al. (2011) reportaron bajo crecimiento para la trucha arcoíris al incluir $50 \%$ de concentrado proteico de papa en la dieta. Xie y Jokumsen (1997) reportaron que el crecimiento de la trucha arcoíris disminuyó conforme se incrementó el nivel de inclusión del concentrado proteico de papa, lo cual puede estar asociado a la baja digestibilidad de AA, tal como se encontró en el presente estudio para camarones. La eficiencia digestiva de AA esenciales en la harina de pescado evaluada en el presente estudio fue alta (97\%), muy por encima de lo reportado por Yang et al. (2009) (87\%), Terrazas-Fierro et al. (2010) (89\%) y Carvalho et al. 2016 (84\%). Esta diferencia puede estar asociada a varios factores, como la especie de pescado, la materia prima (si es un pez entero o subproducto de fileteo, mezcla con vísceras de otras especies, etc.) o el proceso de elaboración (secado con flama directa o con chaqueta de vapor). No obstante, los estudios anteriormente citados no mencionan la materia prima empleada ni el proceso de elaboración.

Llames C, Fontaine J. 1994. Determination of amino acids in feeds: Collaborative study. J. AOAC Int. 77(6): 1362-1402.

Nieto-López M, Tapia-Salazar M, Ricque-Marie D, VillarrealCavazos D, Lemme A, Cruz-Suárez LE. 2011. Digestibility of different wheat products in white shrimp Litopenaeus vannamei juveniles. Aquaculture 319(3-4): 369-376. https://doi.org/10.1016/j.aquaculture.2011.06.046

[NRC] National Research Council [US]. 1983. Nutrient Requirements of Warmwater Fishes and Shellfishes. Washington DC: National Academy Press; 102 pp.

Novus. 1996. Novus Raw Material Compendium. Amino acid profiles database. Brussels (Belgium): [Novus International].

Ozório ROA, Portz L, Borghesi R, Cyrino JEP. 2012. Effects of dietary yeast (Saccharomyces cerevisia) supplementation in practical diets of tilapia (Oreochromis niloticus). Animals 2(1): $16-24$.

https://doi.org/10.3390/ani2010016

Tapia-Salazar M, García-Pérez OD, Velásquez-Soto RA, NietoLópez MG, Villarreal-Cavazos D, Ricque-Marie D, Cruz- 
Suárez LE. 2012. Growth, feed intake, survival, and histological response of white shrimp Litopenaeus vannamei fed diets containing grains naturally contaminated with aflatoxin $=$ Crecimiento, consumo de alimento, supervivencia y respuesta histológica del camarón blanco Litopenaeus vannamei alimentado con dietas de granos contaminados naturalmente con aflatoxinas. Cienc. Mar. 38 (3): 491-504. https://doi.org/10.7773/cm.v38i3.2094

Tecator. 1983. Fat Extraction on Feeds with the Soxtec System HT-The Influence of Sample Preparation and Extraction Media. Application note AN 67/83 (1983.06.13). Sweden: Tecator.

Terrazas-Fierro M, Civera-Cerecedo RC, Ibarra-Martínez L, Goytortúa-Bores E, Herrera-Andrade M, Reyes-Becerra A. 2010. Apparent digestibility of dry matter, protein, and essencial amino acid in marine feedstuffs for juvenile whiteleg shrimp Litipenaeus vannamei. Aquaculture 308 (3-4): 166-173. https://doi.org/10.1016/j.aquaculture.2010.08.021

Tibbets SM, Milley JE, Lall SP. 2006. Apparent protein and energy digestibility of common and alternative feed ingredients by Atlantic cod, Gadus morhua (Linnaeus, 1758). Aquaculture. 261(4): 1314-1327. https://doi.org/10.1016/j.aquaculture.2006.08.052

Tusche K, Berends K, Wuertz S, Susenbeth A, Schulz C. 2011. Evaluation of feed attractants in potato protein concentrate based diets for rainbow trout (Oncorhynchus mykiss). Aquaculture 321 (1-2): 54-60.
Villarreal-Cavazos DA, Cruz-Suárez LE, Tapia-Salazar M, NietoLópez M, Gamboa-Delgado J, Lemme A, Ricque-Marie D. 2017. Effect of feces leaching on apparent digestibility coefficients of Pacific white shrimp (Litopenaeus vannamei) = Efecto de la lixiviación de heces sobre los coeficientes de digestibilidad aparente en camarón blanco del Pacífico (Litopenaeus vannamei). Hidrobiológica 27(3): 353-357.

Villarreal-Cavazos DA, Ricque-Marie D, Peña-Rodríguez A, Nieto-López M, Tapia-Salazar M, Lemme A, Gamboa-Delgado J, Cruz-Suárez LE. 2014. Apparent digestibility of dry matter, crude protein, and amino acids of six rendered by-products in juvenile Penaeus vannamei $=$ Digestibilidad aparente de materia seca, proteína cruda y aminoácidos de seis subproductos de rastro en juveniles de Litopenaeus vannamei. Cienc. Mar. 40(3) 163-172.

http://dx.doi.org/10.7773/cm.v40i3.2427

Xie S, Jokumsen A. 1997. Replacement of fish meal by potato protein concentrate in diets for rainbow trout, Oncorhynchus mykiss (Walbaum): growth, feed utilization and body composition. Aquacult. Nut. 3(1): 65-69. https://doi.org/10.1046/j.1365-2095.1997.00074.x

Yang Q, Zhou X, Zhou Q, Tan B, Chi S, Dong X. 2009. Apparent digestibility of selected feed ingredients for white shrimp Litopenaeus vannamei, Boone. Aquacult. Res. 41(1): 78-86. https://doi.org/10.1111/j.1365-2109.2009.02307.x

Received May 2019, accepted June 2019. 